\title{
TCP/IP Based Intelligent Load Management System in Micro-Grids Network Using MATLAB/Simulink
}

\author{
Muhammad Ali ${ }^{1}$, Muhammad Zakariya ${ }^{2}$, Muhammad Asif $^{3}$, Amjad Ullah $^{2}$ \\ ${ }^{1}$ Electyrical Engineering Department, COMSATS Institute of Information Technology, Abbottabad, Pakistan \\ ${ }^{2}$ Electyrical Engineering Department, NWFP University of Engineering \& Technology, Peshawar, Pakistan \\ ${ }^{3}$ Electyrical Engineering Department, CECOS University of IT, Peshawar, Pakistan \\ Email: mohammad-a@live.com
}

Received May 25, 2012; revised June 28, 2012; accepted July 7, 2012

\begin{abstract}
Computerized power management system with fast and optimal communication network overcomes all major dicrepencies of undue or inadequate load relief that were present in old conventional systems. This paper presents the basic perception and methodology of modern and true intelligent load management scheme in micro grids topology by employing TCP/IP protocol for fast and intelligent switching. The network understudy performs load management and power distribution intelligently in a unified network. Generated power is efficiently distributed among local loads through fast communication system of server in the form of source and clients in the form of loads through TCP/IP. The efficient use of information between server and clients enables to astutely control the load management in a power system of micro grids system. The processing time of above stated system comes out to be $10 \mathrm{~ms}$ faster than others which ensure very less delay as compared to conventional methods. The Micro Grids system operating through TCP/IP control has been implemented in MATLAB/Simulink and results have been verified.
\end{abstract}

Keywords: Intelligent Load Management (ILM); TCP/IP; Micro Grids (MGs); Server and Clients

\section{Introduction}

In power system terminology, when some interdisturbances are leading the overall system towards unstability, the final unwanted solution is load shedding. During load shedding some loads from interconnected network are disconnected for fast and quick recovery of an overall system to its initial settling state. Every grid station has its separate control that controls only the loads in terms of source management. For good management the power generation must be greater than the power consumption then system remains stable. Problems will occur if generation is unable to meet the demands of loads. Then small grids stations are not able to provide energy to their consumers and the only solution is load shedding, to remove power supply from the loads for the system stability.

Conventional schemes were replaced by "Intelligent Load Shedding” [1] scheme which not only has fast response time by introducing TCP/IP for data transfer but manages dropping of specific loads based upon priority and accurately predicts shifts in available generation.

Disturbances of any nature like hurricans, faults, transients etc leads a system towards a fatal scenario and these unwanted actions may take place either on genera- tion side, load side or transmission side depending upon the case. Damir proposed artificial neural network approach for intelligent detection [2] in power systems for stable operation on transmission lines and some issues were also discussed in a report [3] of NERC. Some methods were also suggested for transmission lines control [4] operation. Schemes of load shedding that has been adopted in past have been discussed in power system literature [5-8]. In breaker interlock scheme, when mains breaker was sendining an emergency signal to connected loads breakers all loads were shed without any sequence or priority. However, high priority loads are left untouched during any disturbance and low priority loads are shed from supply. In second method called as under frequency relay scheme that was blind for detecting disturbances but reacted to changes. Slow response time of frequency relays and false dropping out of loads without check were its major discrepencies. Lateron, PLCs System was introduced in an interconnected network but was lacking intelligence due to its blindness to transient shifts and slow response time for breakers to trip in emergency condition. Also, more loads were shed than required. Methods of checking voltage instability and actual loss of transmission capacity $[9,10]$ have not been found. 
The need for local load shedding [11] was proposed by Adibi for an over loaded equipment under certain time constraints. The paper under study has been divided into four main sections illustrating out the work in detail. The first section discusses ILS design using Transmission Control Protocol (TCP/IP), a link that provides intelligent network relation to fast data transfer while the second section relates a LV system (MG) with intelligent control and third section explains the implemented network in MATLAB/Simulink. Finally results are discussed and conclusions with future work have been presented.

\section{ILS Design Using TCP/IP}

In power system each connected network plays an important role either on supply side or generation side or load side .If one connected device becomes problematic than overall system goes towards instability. This is the very important and key feature in power system operation and control because the control and stability are related to each other uncontrolled system is basically unstable. And if that uncontrollable scenario in a power system is not tackled by quick shutdown than heavy blackouts and big disaster may occur in a system. This scene should be avoided because it really affects economic conditions of the organizations that are responsible for its functioning. Here reliability plays a very notable role in a sense that reliable systems are effective and unreliable systems are not stable for very long time. For good and efficient system stability should persists for an unlimited period of time until and unless operating conditions varies drastically. Quick and controlled actions are needed in any system to uproot the changes in controlling parameters which otherwise destroys the systems performance. Otherwise faults occurs in a huge amount and connected equipment becomes faulty It is the scenario where intelligent networks plays important role as traffic control and data transfer is very vital since jamming of information signals will prove fatal.

In Figure 1, block diagram shows the step by step events that will take place in a system quickly. Each block represents an event that has been taking place in a system. First of all power will be produced that will be constant and supplied to the central controller which will make a quick action and send information in form of bits to the local controllers that connected to the respective clients using computational engine. After receiving the useful information by local controllers it has been sent to clients for switching load breakers for an intelligent load shedding. Here all the information has been exchanged through TCP/IP platform because TCP/IP controls transmission, demand, distribution, load management and power in Power systems in sensible way and has

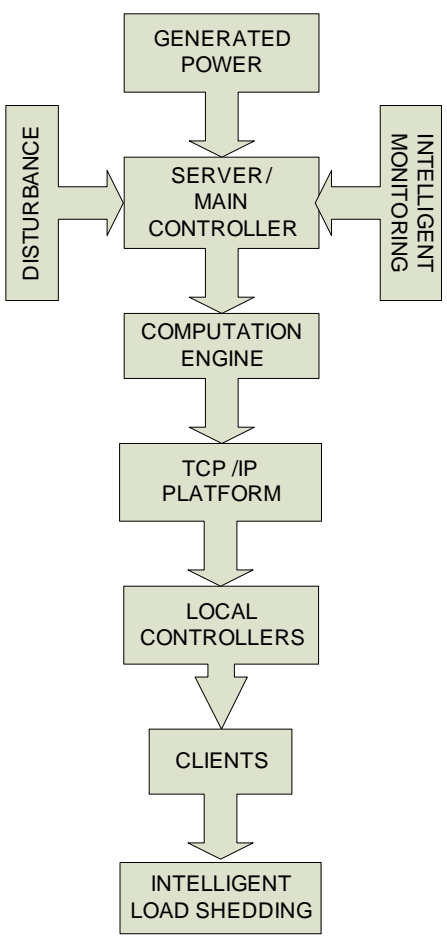

Figure 1. Block diagram of ILS design on TCP/IP platform.

much more recommendations proposed by Holland [12] for shifting electrical networks from SCADA to it. As numbers of system may be interconnected and they might sent data and information to each other so the system should be strong to hold such a heavy traffic and should be manage from not to get mal functional. As controller handles large amount of data and update it rapidly after mille second so it not easy for it to make decisions of each control and emergency signal at that specific time intervals. And delay can make Power system gets unstable. Therefore for the data transferring reliable medium should be used, having large band width and speed. Channel response should be fast too. For this to be accomplished transmission control protocol is prior for interconnected power system which not only provides high band width but also much quick in response. Secondly, it is connection oriented protocol and data traffic remains secure and safe.

In the Figure 2 shown above which is a server-client model and information that has been transmitted on TCP platform. Server is the central controller having a data base and clients are basically loads here. Local Ethernet networking has been extended there. Communication between both parties is really fast and controlled intelligently by server.

\section{MG's Network with Intelligent Control}

Micro Grids are the small networks [13] with either renewable energy resources or generating sources connected 


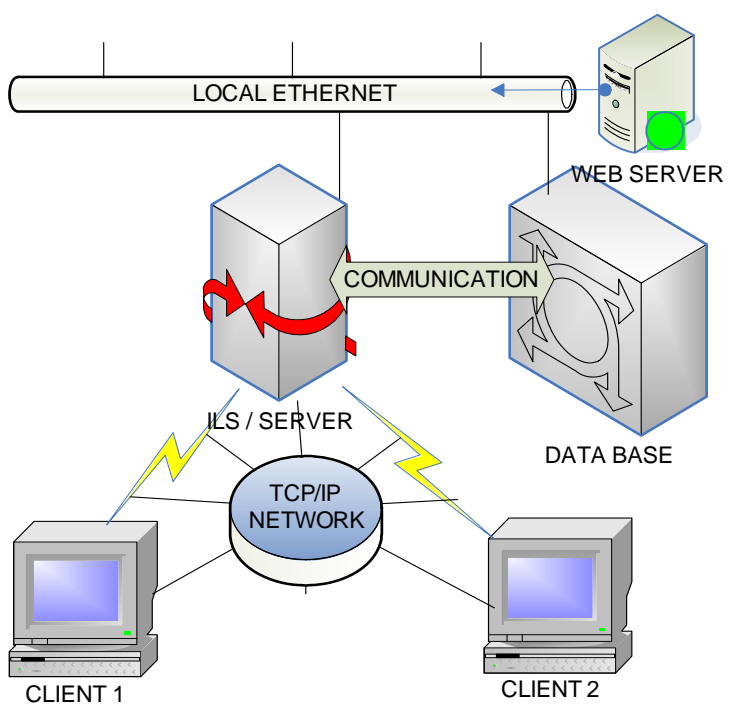

Figure 2. Server-client intelligent network model design.

to it in a controlled fashion. The non-autonomous form of MG has much increased reliability than stand alone MG. It has a central controller called as micro grid central controller which has a strong communication network with its local controllers as clients. Useful information between both the parties is carried through TCP platform. MG connected with utility performs stable op- erations and quality of power is delivered to consumers. Several issues regarding micro grids [14] have been discussed in detail. Figure $\mathbf{3}$ shows an intelligent control network of MG's in server-client scenario.

Simple model of a MG network that has taken as base for intelligent control implementation is shown in Figure 4 with one generation source and two clients as constant loads. As micro grids contains many generation sources and different kinds of loads connected to it so each load has its own controller which sends signals to main controller for making of decisions in quick succession. In such type of systems, a LAN is mainly obtained and if many micro grids are connected to each other than wide area network is obtained to control all the connected system working well. Nowadays, different power system applications are following this protocol as a leading way of communication between local controllers and main controller. e.g., wind farms, local factories, industries, hydal generation systems etc all are trying to shift previous systems to TCP/IP networking embedded inside each operating instrument. In modern terminology if path communication is between controllers and every operating load or instrument than it is called intelligent control response [15] in micro grids because stable operation requires quick control actions in its operation. Simplified micro grid network is shown in Figure 5.

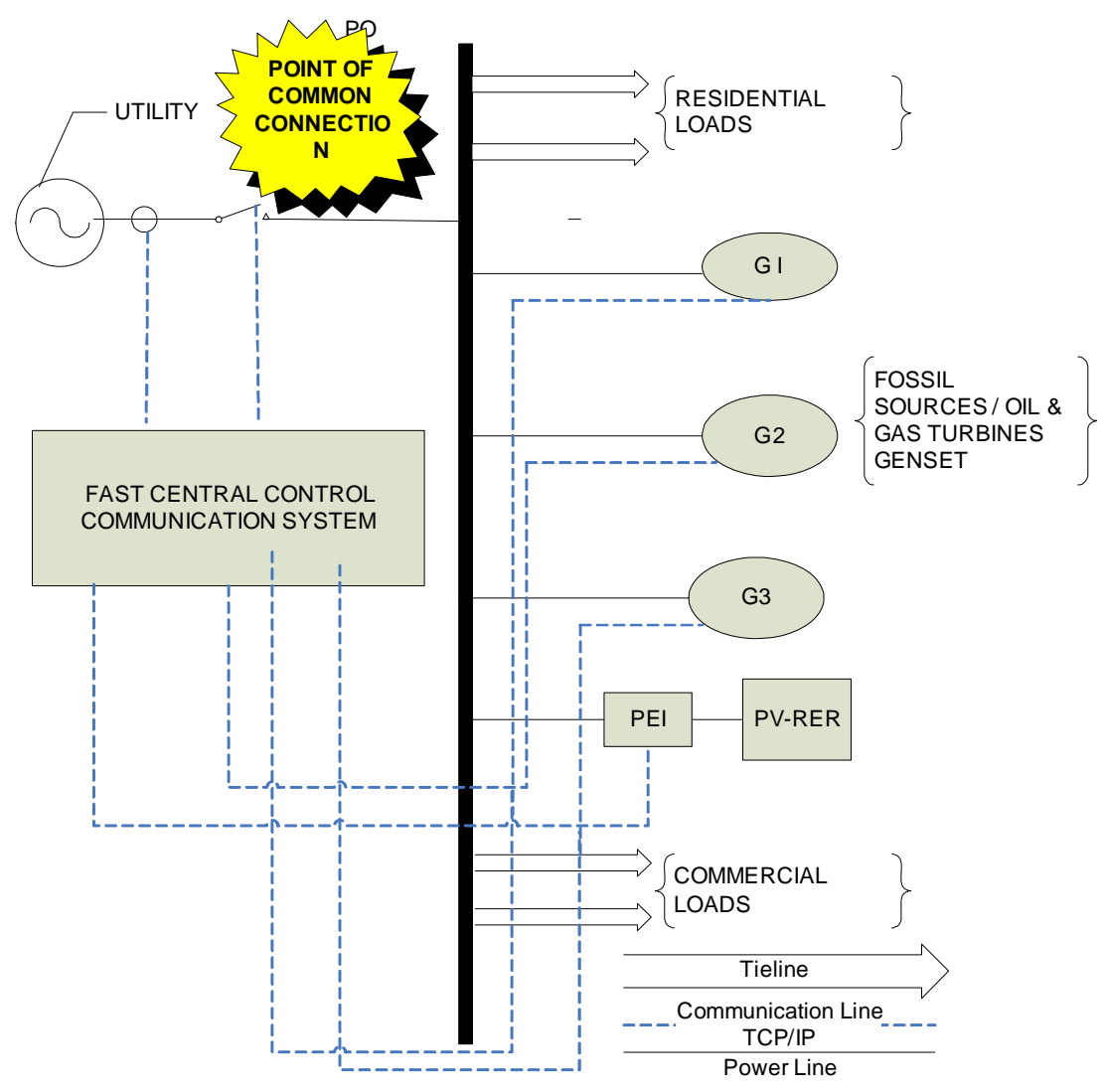

Figure 3. MG in intelligent control scenario. 


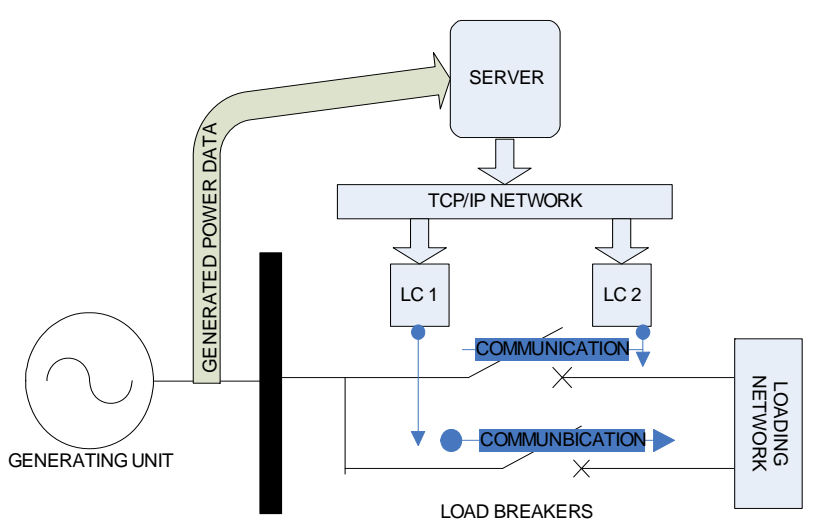

Figure 4. Power system implemented network architecture.

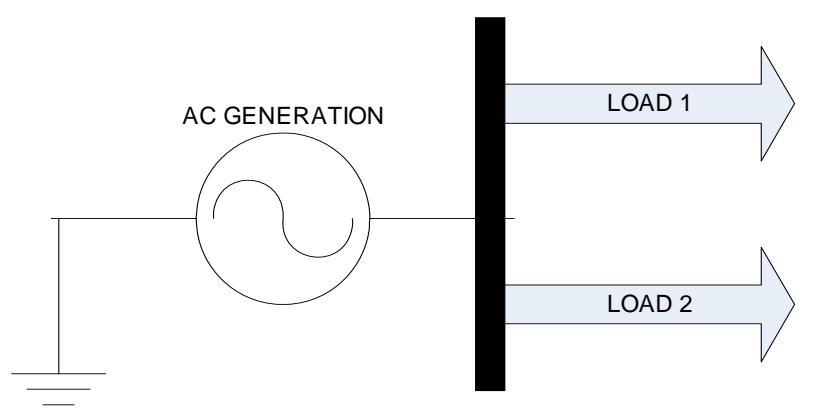

Figure 5. MG simplified model.

\section{Implemented Network in MATLAB}

In this article, two controllers have been defined, one is main controller acting as a server which is primary one and the other one is secondary which acts as client. TCP/IP networking channel is wide spread on the whole network. Loads to be shed are decided by server and this useful information is carried to clients which has its own local controllers through TCP as shown in Figure 6. Priority of loads has been defined in the server and quick decisions of ILS are carried out with the help of TCP/IP.

Final system that has been implemented in MATLAB/ Simulink is shown in Figure 7. To precede that very basic idea of ILS, the work basically comprises of four major systems. And these networks were made in MATLAB/Simulink. First of all the very basic system was a manual control network that has been developed in SIMULINK and two controllers with defined programming distributing generated power actually between two loads was discussed. Generated power was manually controlled and loads were connected and disconnected according to defined priority. Switching of loads with power available and defined priority is shown in Table 1.

First system was replaced by second one in the automated control topology. In this case the produced power

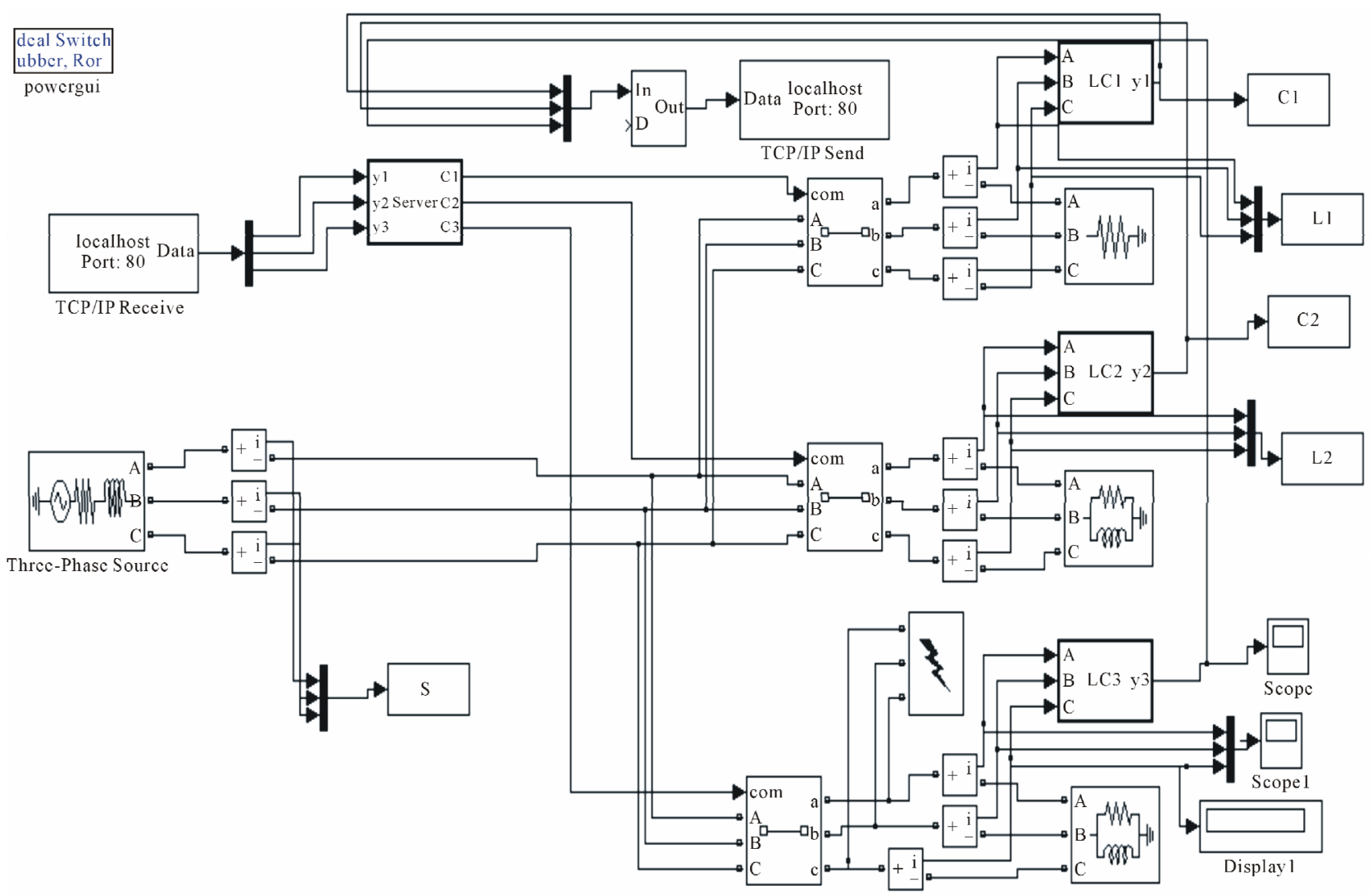

Figure 6. Implemented network in MATLAB. 


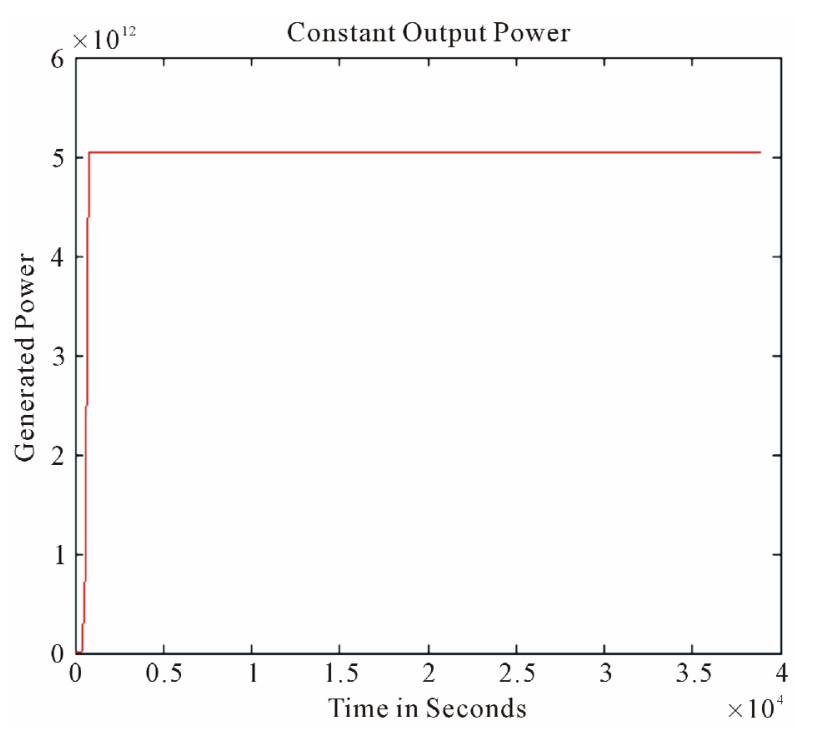

Figure 7. MG power delivered to server.

Table 1. Switching at defined thresholds of power generated.

\begin{tabular}{cccccc}
\hline Volts & $\begin{array}{c}\text { Generated } \\
\text { Power } \\
\text { "Watts" }\end{array}$ & $\begin{array}{c}\text { Decision Criteria } \\
\text { of Server }\end{array}$ & $\begin{array}{c}\text { Data Sent } \\
\text { from Sever }\end{array}$ & \multicolumn{2}{c}{$\begin{array}{c}\text { Data Received } \\
\text { by Clients }\end{array}$} \\
\cline { 5 - 7 } $25 \mathrm{KV}$ & $5 \mathrm{E} 12 \mathrm{~W}$ & $\mathrm{Pw}>=5 \mathrm{E} 12$ & $3(11)$ & 1 & 1 \\
$2500 \mathrm{~V}$ & $2.5 \mathrm{E} 4 \mathrm{~W}$ & $\begin{array}{c}\mathrm{Pw}<5 \mathrm{E} 12 \& \\
\mathrm{pw}>=2.5 \mathrm{E} 4\end{array}$ & $2(10)$ & 1 & 0 \\
$250 \mathrm{~V}$ & $2.5 \mathrm{E} 2 \mathrm{~W}$ & $\begin{array}{c}\mathrm{Pw}<2.5 \mathrm{E} 4 \& \\
\mathrm{pw}>=2.5 \mathrm{E} 2\end{array}$ & $1(01)$ & 0 & 1 \\
$<250 \mathrm{~V}$ & & & $0(00)$ & 0 & 0 \\
\hline
\end{tabular}

was automated made constant in a steady state conditions and then managed according to supply. In that particular scheme, constant power was handled through automated system. Using binary number system, loads were operated. Third system was built on the bases of automation with improved concept of data read and data write with same controller options using temporary memory storage option. Then finally the fourth system was very improved form of all of them. There were four controllers that were built. One was acting as server whiles other three as local load controller acting as clients.

The useful and important information was shared dynamically using transmission control protocol. Using this platform, for fast traffic delivery, control decisions were taken. As the load management was done automated through TCP/IP plat form through intelligent controllers, so scheme of ILS was developed in MATLAB. Previous system that power system engineers developed like breaker interlock technique, under frequency decay technique and PLC's operating systems have major disadvantage of slow response time for the breakers to operate.
Using TCP/IP plat form for fast data transfer, the overall processing time that came out was 10 mille second.

\section{Simulation and Results}

The Figure 7 explains the nature of power produced from the generating source which is constant since the system can only accept the constant output because it will not make accurate decisions on the basis of varying power. It also shows that it takes a very small time to for constant output being produced. If the source voltage magnitudes are to be varied than output will also vary in the same manner. Figure 8 shows the information generated by server to clients.

Figure 9 shows the information delivered by the server to respected clients which are acting as loads.

Above Figure 8 shows that $10 \mathrm{~ms}$ is the total processing time taken for the data to reach from the server to clients and finally to load breakers through TCP/IP platform. One " 1 " signal is received at $10 \mathrm{~ms}$ second so

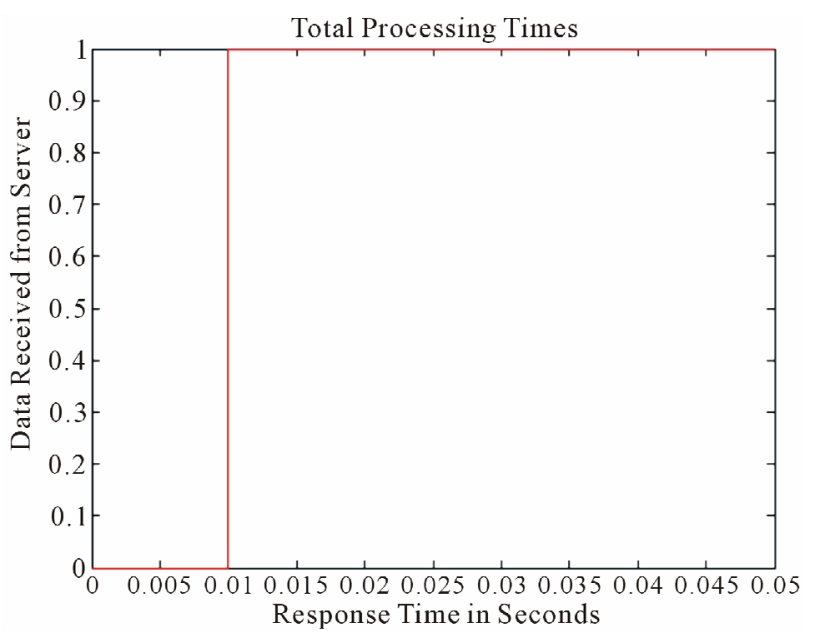

Figure 8. Total processing time.

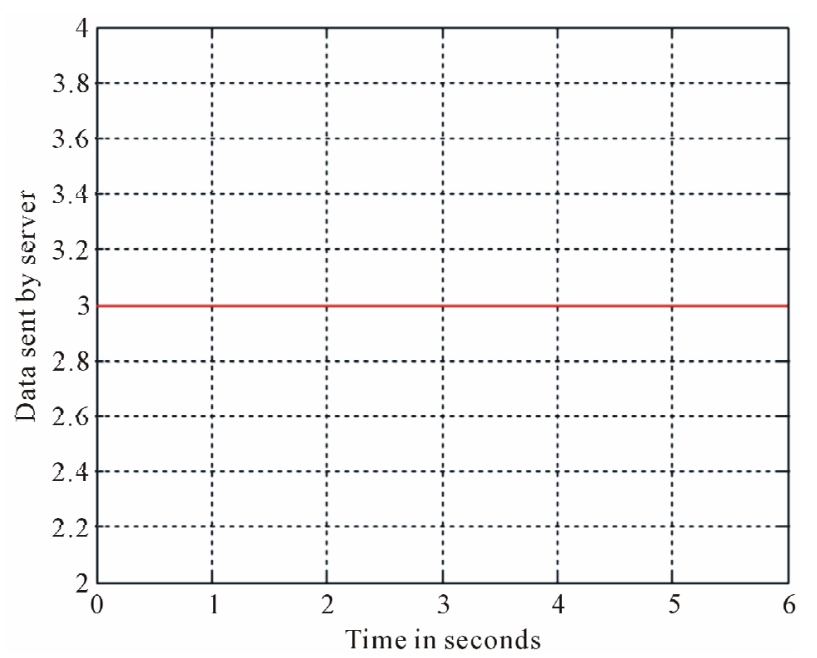

Figure 9. Information delivered by server. 
breaker at that position will remain closed and load connected to it will function normally.

Below Figures 10 and $\mathbf{1 1}$ shows a current and voltage signal flowing through the load breaker after it receives a control signal and after that faulty line will be cleared for that connected breaker. So the systems become intelligent when the responses are quick, fast but accurate according to the desired condition. Intelligence lies in the fact that system responds only to true directed states.

\section{Conclusion}

Intelligent load management systems are needed every where either in power systems, communication systems etc because without them sometimes there are always a chance that information is miss interpreted and unwanted events occur. Intelligence of a system basically lies in a controller which is the brain of a system which monitors and controls the whole network. In power systems especially when supply is insufficient to meet the demand of connected loads the intelligence lies in a fact that which loads are shifted either in on or off position.

\section{Future Work}

Since the latest trends are now from micro grids to smart grids which is a big change and development in power industry. As the smart grid concept includes control up to

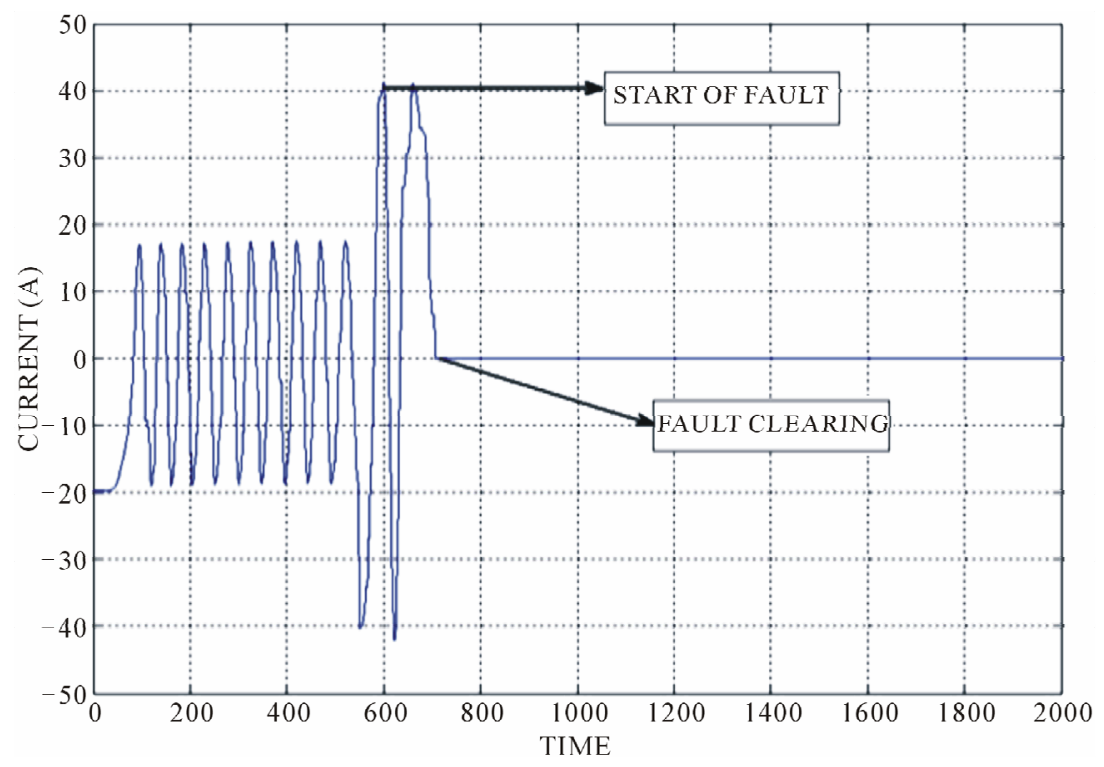

Figure 10. Load breakers response after receiving current control signal.

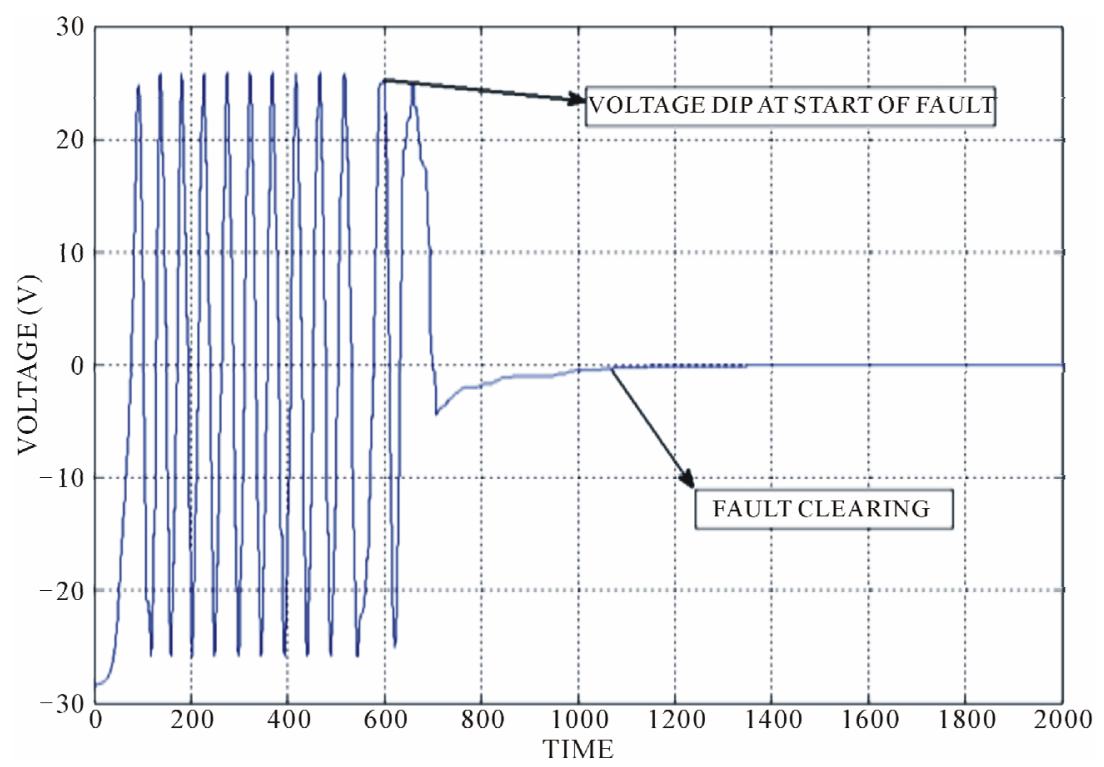

Figure 11. Load breakers response after receiving voltage control signal. 
the instrument level, so if the instrument corresponds and communicates intelligently and accurately so all previous problems will be smoothened out in near future. The concept of TCP/IP brings evolution in a system whenever there is a huge interconnected traffic involved. Also Smart grids technology will be flourished and polished by it. A lot of work is still going on in this new area and will continue in new future.

\section{Acknowledgements}

The special thank goes to Mr. Waheed-ur-Rehaman who supported us in providing help in the preparation regarding to this article. We are also thankful to Mr. Dr. A. Rashid he has made a great contribution throughout our work especially in editing the text and paper formatting.

\section{REFERENCES}

[1] F. Shookh and J. J. Dai, “An ILS System Application in Large Industrial Facility,” IAS 2005.

[2] L. H. Fink, D. E. Badley, J. E. Koehler, D. A. McInnis, and J. J. Redmond, "Emergency Control Practices," Transactions on PAS, Vol. 104, 1985, pp. 2336-2441.

[3] North American Electric Reliability Council, “1988: System Disturbance,” NERC Report, 1989.

[4] L. O. Barthold, "Technical Limits to Transmission System Operation,” EPRI EL-5859, Final Report, 1988.

[5] A. Maiorano, R. Sbrizzai, F. Torelli and M. Trovato, "Intelligent Load Shedding Schemes for Industrial Customers with Cogeneration Facilities," Power Engineering Society 1999 Winter Meeting, IEEE, Vol. 2, 1999, pp. 925930.
[6] D. Novosel and R. L. King, "Using Artificial Neural Networks for Load Shedding to Alleviate Overloaded Lines," IEEE Transactions on Power Delivery, Vol. 9, No. 1, 1994, pp. 425-433. doi:10.1109/61.277714

[7] L. J. Shih, W. J. Lee, J. C. Gu and Y. H. Moon, “Application of $\mathrm{df} / \mathrm{dt}$ in Power System Protection and Its Implementation in Micro Controller Based Intelligent Load Shedding Relay," Industrial and Commercial Power Systems Technical Conference, Memphis, 6-9 May 1991.

[8] W. Lee and J. C. Gu, “A Micro Computer-Based Intelligent Load Shedding Relay," IEEE Transactions on Power Delivery, Vol. 4, No. 4, 1989, pp. 2018-2024. doi:10.1109/61.35626

[9] System Protection Schemes in Power Networks, CIGRE Technical Report No. 187, 2001.

[10] C. W. Taylor, "Power System Voltage Stability,” 1994, Undervoltage Load Shedding Guidelines, Western Electricity Coordinating Council, Salt Lake City, 1999.

[11] M. M. Adibi and D. K. Thome, "Local Load Shedding," IEEE Transaction on PWRS, Vol. 3, No. 3, 1988, pp. 1220-1229.

[12] K.-H. Mak and B. Holland, "Migrating Electric power networks from SCADA to TCP/IP," Power Engineering Journal, Vol. 16, No. 6, 2002, pp. 305-331. doi:10.1049/pe:20020604

[13] Micro Grids Workgroup, "Large Scale Integration of Micro Generation to Low Voltage Grids Target Action 1: Annex 1,” Description of Work, Athens, 2002.

[14] D. Pudjianto and G. Strbac, "Investigation of Regulatory, Commercial, Economic and Environmental Issues in Micro Grids,” EU Research Project 2002-2006.

[15] R. H. Lasseter, "Control and Design of Micro Grid Components,” PSERC Final Report, 2007. 